\title{
Effects of Spin Pumping on Spin Waves in Antiferromagnetically Exchange-Coupled Double Layers with Surface Anisotropy
}

\author{
P. BALÁZ̆ ${ }^{a, b, *}$ AND J. BARNAŚ $\widehat{S}^{a, b}$ \\ ${ }^{a}$ A. Mickiewicz University, Faculty of Physics, Umultowska 85, 61-614 Poznań, Poland \\ ${ }^{b}$ Institute of Molecular Physics, Polish Academy of Sciences, M. Smoluchowskiego 17, 60-179 Poznań, Poland

\begin{abstract}
Spin wave modes in antiferromagnetically exchange-coupled magnetic double layers are analyzed theoretically.
\end{abstract} \\ The considered structure is assumed to be covered by a nonmagnetic metallic layer. The spin wave frequencies \\ and spin wave life times are determined from the macroscopic description based on the Landau-Lifshitz-Gilbert \\ equation, which includes the torque due to spin pumping to the cap layer.
}

DOI: 10.12693 /APhysPolA.128.150

PACS: 75.40.Gb, 75.76.+j, 75.78.-n

\section{Introduction}

Spin waves in artificially layered magnetic structures were extensively studied two decades ago $[1,2]$. Of particular interest were spin waves in exchange-coupled double layers, where two metallic magnetic films were separated by a metallic nonmagnetic spacer [3-5]. The two magnetic films in such structures are coupled via indirect exchange interaction of RKKY type, which oscillates between ferromagnetic and antiferromagnetic with increasing thickness of the spacing layer [6]. The exchange interaction was shown to significantly modify the corresponding spin wave spectra $[3-5,7]$, which in turn were used to determine the interlayer exchange coupling parameter.

Recent interest in spin waves is associated, among others, with spin currents inherently accompanying spin waves, and with such phenomena like spin transfer torque and spin pumping $[8,9]$. In the latter case, a spin current is pumped through a ferromagnet/nonmagnet interface by precessing magnetic moment of the ferromagnetic layer $[10,11]$. In this paper we consider the influence of spin pumping on the spin wave spectra in antiferromagnetically coupled magnetic double layers with perpendicular surface anisotropy. For simplicity, we assume that the spin pumping and surface anisotropy occur only at the top interface of the studied structure, see Fig. 1. By generalizing the macroscopic approach by Vohl et al. [3], we calculate the spin-wave eigenfrequencies and the related spin-wave life times.

\section{Model}

Figure 1 shows a magnetic structure under consideration, which consists of two magnetic layers $F_{j}(j=1,2)$ separated by a nonmagnetic spacer. The structure is deposited on a nonmetallic substrate and is covered by a

* corresponding author; e-mail: balaz@ifmpan.poznan.pl metallic cap layer. We assume that magnetizations of both films in the ground state are antiparallel due to antiferromagnetic interlayer exchange coupling, and are parallel to the easy axis ( $\pm z$-axis $)$.

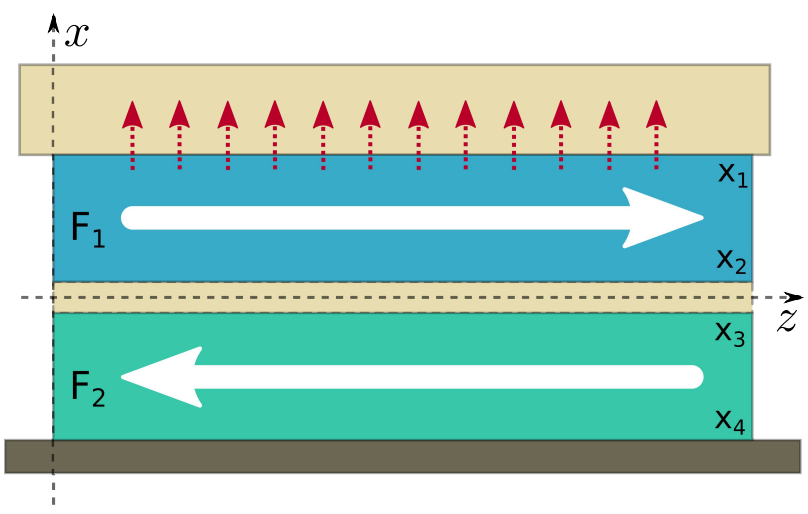

Fig. 1. Scheme of the magnetic double layer with antiparallel alignment of magnetic moments. Two magnetic insulating layers, $F_{j}$, distinguished with the layer index $j$ ( $j=1$ for the top and $j=2$ for the bottom layers) are separated by a thin nonmagnetic metallic spacer layer. The external interfaces are at $x_{1}$ and $x_{4}$, while the internal interfaces are at $x_{2}$ and $x_{3}$, as indicated. The red (dotted) arrows represent the spin pumping current.

Internal dynamics of the $j$-th magnetic layer $(j=$ 1,2 ) is described by the Landau-Lifshitz-Gilbert (LLG) equation

$$
\frac{\mathrm{d} \boldsymbol{M}_{j}}{\mathrm{~d} t}=-\gamma_{j} \mu_{0} \boldsymbol{M}_{j} \times \boldsymbol{H}_{\mathrm{eff} j}+\frac{\alpha_{j}}{M_{\mathrm{s} j}} \boldsymbol{M}_{j} \times \frac{\mathrm{d} \boldsymbol{M}_{j}}{\mathrm{~d} t},
$$

where $\boldsymbol{M}_{j}=\boldsymbol{M}_{j}(\boldsymbol{r}, t)$ is the magnetization of the $j$-th layer, $M_{\mathrm{s} j}=\left|\boldsymbol{M}_{j}\right|$ is the corresponding saturation magnetization, $\alpha_{j}$ is the Gilbert damping parameter, $\mu_{0}$ is the vacuum permeability, and $\gamma_{j}=|e| g_{j} / 2 m$, with $e$ and $m$ being the electron charge and electron mass, respectively, and $g_{j}$ denoting the Lande factor for the $j$-th layer. Moreover, $\boldsymbol{H}_{\text {eff } j}=\boldsymbol{H}_{\text {eff } j}(\boldsymbol{r}, t)$ stands for an effective magnetic 
field in the $j$-th layer

$$
\begin{gathered}
\boldsymbol{H}_{\mathrm{eff} j}(\boldsymbol{r}, t)=\frac{H_{\mathrm{a} j}}{M_{\mathrm{s} j}}\left[\boldsymbol{M}_{j}(\boldsymbol{r}, t) \cdot \hat{\boldsymbol{e}}_{z}\right] \hat{\boldsymbol{e}}_{z}+\boldsymbol{h}_{j}(\boldsymbol{r}, t) \\
+\frac{2 A_{j}}{\mu_{0} M_{\mathrm{s} j}^{2}} \nabla^{2} \boldsymbol{M}_{j}(\boldsymbol{r}, t),
\end{gathered}
$$

where $H_{\mathrm{a} j}$ and $\boldsymbol{h}_{j}$ are the anisotropy and dipolar fields, respectively, while the latter term describes the intralayer exchange interaction, with the exchange stiffness parameter $A_{j}$.

We restrict considerations to the Voigt geometry, where spin waves propagate perpendicularly to the magnetization orientation. Let us assume plane-wave solutions of frequency $\boldsymbol{\omega}$ and wave vector $\boldsymbol{q}=\left(q_{x}, q_{y}\right)$, and define the vector $\boldsymbol{\xi}_{j}(\boldsymbol{r})=\left[m_{j, x}(\boldsymbol{r}), m_{j, y}(\boldsymbol{r}), \psi_{j}(\boldsymbol{r})\right]$, consisting of the dynamical magnetization components, $m_{j, x}(\boldsymbol{r})$ and $m_{j, y}(\boldsymbol{r})$, and the scalar potential $\psi_{j}(\boldsymbol{r})$ defining the dipolar field $\boldsymbol{h}_{j}(\boldsymbol{r})$ via the magnetostatic equations, $\boldsymbol{h}_{j}=-\nabla \psi_{j}(\boldsymbol{r})$. A general solution for the $n$-th component $(n \in\{1,2,3\})$ of $\boldsymbol{\xi}_{j}(\boldsymbol{r})$ can be written in the form [3]:

$$
\xi_{j, n}=\sum_{l=1}^{3}\left[C_{j, n}^{(l)} \cos \left(k_{j, l} x\right)+D_{j, n}^{(l)} \sin \left(k_{j, l} x\right)\right] \mathrm{e}^{\mathrm{i} \boldsymbol{q} \cdot \boldsymbol{\rho}}
$$

where $\boldsymbol{\rho}=(x, y)$, and

$$
\begin{aligned}
& k_{j, 1}^{2}=-q_{y}^{2}, \\
& k_{j, 2(3)}^{2}=-\frac{1}{Q_{j}}\left[1 / 2+\frac{H_{\mathrm{a} j}}{M_{\mathrm{s} j}}-\mathrm{i} \alpha_{j} f_{j} \pm \sqrt{f_{j}^{2}+(1 / 2)^{2}}\right] \\
& -q_{y}^{2} .
\end{aligned}
$$

Here, $f_{j}=\omega / \omega_{M, j}, \omega_{M, j}=\gamma_{j} \mu_{0} M_{\mathrm{s} j}$, and $Q_{j}=$ $2 A_{j} /\left(\mu_{0} M_{\mathrm{s} j}^{2}\right)$. For each $j$ only 6 parameters, $C_{j, 1}^{(l)}$ and $D_{j, 1}^{(l)}$ for $l=1,2,3$, are independent. These parameters can be determined from appropriate boundary conditions at the four interfaces.

At the top external interface, located at $x=x_{1}$, we apply the Rado-Weertman [12] boundary conditions, generalized by including the effects due to spin pumping

$$
\begin{aligned}
& \left.\left(A_{1} \frac{\partial}{\partial x}-\mathrm{i} G_{1} \omega\right) m_{1, y}\right|_{x=x_{1}}=0, \\
& \left.\left(A_{1} \frac{\partial}{\partial x}-\mathrm{i} G_{1} \omega-K_{1}^{s}\right) m_{1, x}\right|_{x=x_{1}}=0,
\end{aligned}
$$

where $G_{1}=\hbar g_{\mathrm{r} 1} /(8 \pi)$, with $g_{\mathrm{r} 1}$ being the real part of the mixing conductance of the top interface, normalized to $e^{2} / h$, while $K_{1}^{s}$ is the perpendicular anisotropy constant at the top interface. The boundary conditions for the bottom interface are similar to those given by Eqs. (5), but with zero surface anisotropy and vanishing mixing conductance. At the internal interfaces, in turn, we use the standard Hoffmann boundary conditions [13]. In addition, the tangential component of $\boldsymbol{h}_{j}(\boldsymbol{r})$ and the normal component of $\boldsymbol{h}(\boldsymbol{r})+\boldsymbol{m}(\boldsymbol{r})$ must be continuous across the interfaces, which leads to four additional boundary conditions as described in Ref. [3]. Equations (5) together with the Hoffmann and magnetostatic conditions form the complete set of 12 boundary conditions, which have to be fulfilled by the magnetization and dipolar field. These equations have solutions when the corresponding determinant vanishes, and this condition is obeyed for specific values of the frequency $\omega$, which are the spinwave eigenfrequencies.

\section{Results}

The main objective of the analysis is a detailed description of the influence of spin pumping at the top interface on the spin wave frequency and damping. For both magnetic films we assume parameters typical of yttrium-irongarnets (YIG) [14], i.e. $A_{j}=4.17 \times 10^{-12} \mathrm{~J} \mathrm{~m}^{-1}, M_{\mathrm{s} j}=$ $1.56 \times 10^{5} \mathrm{~A} \mathrm{~m}^{-1}, K_{j}^{s}=10^{-4} \mathrm{~J} / \mathrm{m}^{2}, \alpha_{j}=6.7 \times 10^{-5}$, and $g_{j}=2$ (for $\left.j=1,2\right)$. For the thickness of the magnetic layers we assume $L_{1}=L_{2} \equiv L=100 \mathrm{~nm}$. In turn, thickness of the spacing layer is assumed to be much smaller. This thickness determines the interlayer exchange coupling, and thus it is effectively included in the calculations via the interlayer exchange parameter $A_{12}$. Although, the dynamic coupling between the two magnetic layers via the spin pumping is possible, we do not address this problem here and assume that the mixing conductance of the nonmagnetic spacer is negligible.

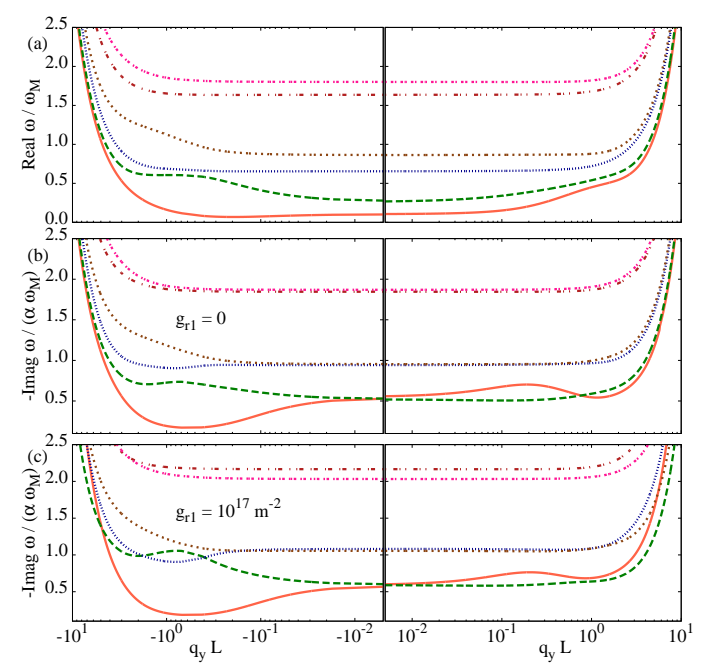

Fig. 2. Spin waves eigenfrequencies in the antiparallel magnetic configuration as a function of $q_{y} L$, calculated for $A_{12}=-5 \times 10^{-4} \mathrm{~J} \mathrm{~m}^{-2}$ and in-plane uniaxial bulk anisotropy $H_{\mathrm{a}}=10^{-2} M_{\mathrm{s}}$. (a) real part of the frequency $\omega,(b)$ imaginary part of $\omega$ in the case of no spin pumping, (c) imaginary part of $\omega$ in the case of spin pumping at the top interface, $g_{\mathrm{r}_{1}}=10^{17} \mathrm{~m}^{-2}$.

Figure 2 shows the real and imaginary parts of the spin wave eigenfrequency as a function of the wave vector $q_{y}$ for the lowest six modes and for two opposite propagation orientations. The corresponding mode profiles, calculated for $q_{y} L=0.1$, are shown in Fig. 3. Here $n$ is the mode index, with $n=1$ corresponding to the lowest mode and increasing with increasing mode frequency. The first two modes have mixed surface-bulk character. The higher modes contain much less interfacial contribution. The first mode is localized at the bottom interface 

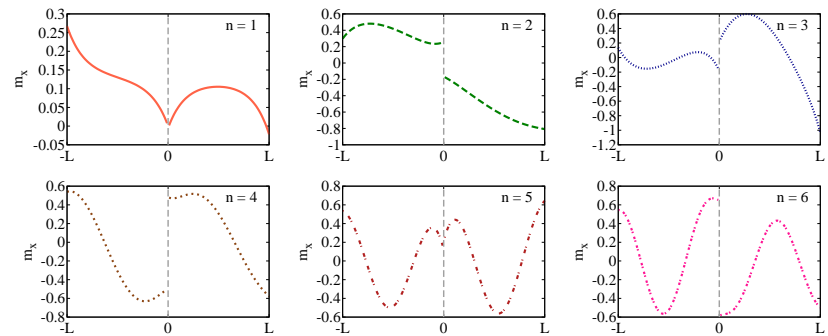

Fig. 3. Mode profiles corresponding to Fig. 2 for $q_{y} L=0.1$.

and almost vanishes at all others interfaces, while the modes corresponding to $n=2,3$ are more pronounced at the top interface and remain nonzero also at the internal interfaces. Therefore, these two modes are particularly influenced by the spin pumping at the top interface.
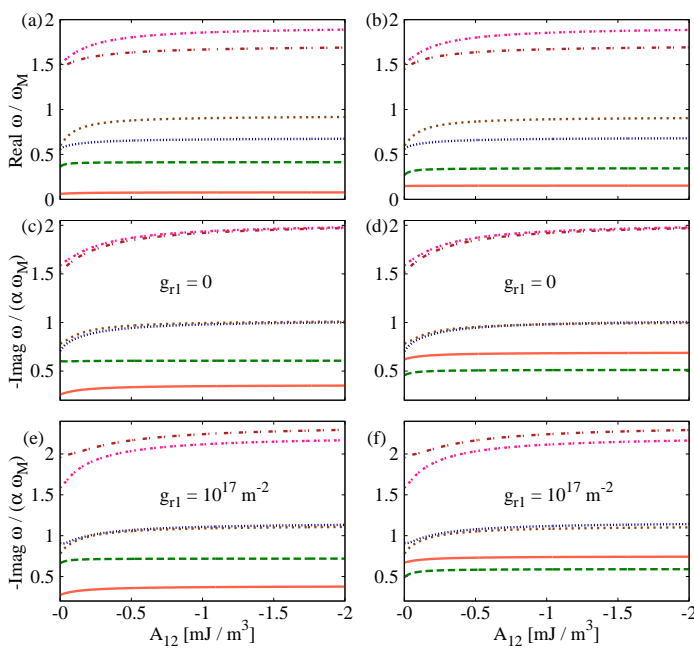

Fig. 4. Spin waves eigenfrequencies in the antiparallel configuration as a function of $A_{12}$ for $q_{y} L=-0.1$ (left column) and $q_{y} L=0.1$ (right column). (a,b) Real parts of frequency $\omega,(\mathrm{c}, \mathrm{d})$ imaginary parts of $\omega$ in the case of no spin pumping, (e,f) imaginary parts of $\omega$ in the case of spin pumping at the top interface, $g_{\mathrm{r} 1}=10^{17} \mathrm{~m}^{-2}$. Other parameters as in Fig. 2.

The interface anisotropy changes separation of the modes marked with $n=2$ and $n=3$, especially for $q_{y} L$ close to -1 . This can be seen when comparing the spectrum in Fig. 2 with the corresponding one in the absence of anisotropy (not shown). Apart from this, the real parts of the eigenfrequencies reveal nonreciprocality of the surface mode propagation, i.e. the mode frequencies for $q_{y}>0$ and $q_{y}<0$ are different. This difference depends on the surface mode contribution to a particular eigenmode, and disappears for pure bulk modes. The spin wave nonreciprocality is visible also in the imaginary parts of the eigenfrequencies, Fig. 2b,c.

The imaginary parts of the eigenfrequencies determine the spin wave life time. These life times are remarkably influenced by the spin pumping, as follows from Fig. $2 \mathrm{~b}$ and $c$, which show the imaginary parts of $\omega$ in the absence and presence of the spin pumping at the top interface, respectively. Generally, spin pumping enhances the spin wave damping, while its influence on the real parts of $\omega$ is less important.

Variation of the spin wave eigenfrequencies with the interlayer exchange parameter $A_{12}$ is shown explicitly in Fig. 4. The nonreciprocality of surface mode propagation is also clearly seen, when comparing left (for $q_{y} L=-0.1$ ) and right (for $q_{y} L=0.1$ ) parts of Fig. 4 . Both real and imaginary parts of the eigenfrequencies vary with $A_{12}$ at small values of the interlayer coupling, and then quickly saturate with increasing $A_{12}$.

\section{Summary}

We have analyzed the influence of spin pumping on the spin wave modes propagating in a magnetic double layer with antiferromagnetic interlayer exchange-coupling and surface anisotropy. The spin pumping is shown to enhance spin wave damping, while it has rather weak influence on the real parts of eigenfrequencies for the considered system. The modes propagating in the opposite directions may have significantly different frequencies and life times, provided they contain some contribution of surface modes due to hybridization of the bulk and surface waves.

\section{Acknowledgments}

This work was carried out within the Project NANOSPIN PSPB-045/2010 supported by a grant from Switzerland through the Swiss Contribution to the enlarged European Union.

\section{References}

[1] P. Grunberg, Prog. Surf. Sci. 18, 1 (1985).

[2] G.T. Rado, R.J. Hicken, J. Appl. Phys. 63, 3885 (1988).

[3] M. Vohl, J. Barnaś, P. Grünberg, Phys. Rev. B 39 , 12003 (1989).

[4] J. Barnaś, P. Grünberg, J. Magn. Magn. Mater. 82, 186 (1989).

[5] B. Hillebrands, Phys. Rev. B 37, 9885 (1988).

[6] S.S.P. Parkin, N. More, K.P. Roche, Phys. Rev. Lett. 64, 2304 (1990).

[7] J. Fassbender, F. Nortemann, R.L. Stamps, R.E. Camley, B. Hillebrands, G. Güntherodt, S.S.P. Parkin, Phys. Rev. B 46, 5810 (1992).

[8] R. Cheng, J. Xiao, Q. Niu, A. Brataas, Phys. Rev. Lett. 113, 057601 (2014).

[9] H. Skarsvåg, A. Kapelrud, A. Brataas, Phys. Rev. B 90, 094418 (2014)

[10] Y. Tserkovnyak, A. Brataas, G.E.W. Bauer, Phys. Rev. Lett. 88, 117601 (2002).

[11] Y. Tserkovnyak, A. Brataas, G.E.W. Bauer, B.I. Halperin, Rev. Mod. Phys. 77, 1375 (2005).

[12] G.T. Rado, J. Weertman, J. Phys. Chem. Solids 11, 315 (1959).

[13] F. Hoffmann, A. Stankoff, H. Pascard, J. Appl. Phys. 41, 1022 (1970).

[14] Y. Zhou, H.J. Jiao, Y.T. Chen, G.E.W. Bauer, J. Xiao, Phys. Rev. B 88, 184403 (2013). 